\title{
Pneumonien verhindern
}

\section{Serie Prophylaxen - Teil 4 Für Pflegende gehört prophylaktisches Arbeiten zum beruf- lichen Selbstverständnis. Es geht darum, Risiken für gesundheitliche Beeinträchtigungen zu erkennen, Symptome richtig zu bewerten und kompetent vorzubeugen.}

$\mathbf{P}$ neumonien sind in Deutschland die am häufigsten tödlich verlaufenden Infektionskrankheiten. Es werden mehr Menschen mit einer Pneumonie in die Klinik eingewiesen als mit Herzinfarkt oder Schlaganfall. In den westlichen Industrieländern ist die nosokomiale Pneumonie die zweithäufigste Hospitalinfektion. Aufgrund der negativen Beeinflussung des Genesungsprozesses des Patienten nimmt die Pneumonie innerhalb der stationären Versorgung einen hohen Stellenwert ein. Somit ist es im Rahmen einer ganzheitlichen Patientenversorgung unerlässlich, Möglichkeiten der Pneumonieprophylaxe zu kennen und in einem multiprofessionellen Team konsequent anzuwenden.

Eine Pneumonie wird durch eine Entzündung des Lungenparenchyms, welche durch allergische, physikalisch-chemische oder infektiöse Ursachen ausgelöst wird, definiert. Die Pneumonie wird (nach der Genese) in folgende Arten eingeteilt:

- Typische und atypische Pneumonien aufgrund der auslösenden Erreger

- Ambulante und nosokomiale Pneumonien aufgrund des Auftretens

- Primäre und sekundäre Pneumonien aufgrund der primären Erkrankung

\section{Symptome und Risikofaktoren}

$\mathrm{Zu}$ Beginn ist eine Pneumonie durch hohes Fieber, Husten und meist eitriges Sputum gekennzeichnet. Zusätzlich treten oftmals eine schmerzhafte Atmung in Verbindung mit Schonatmung, Dyspnoe, mit eventueller Zyanose und Tachykardie auf.

Der Entstehung einer Pneumonie liegen verschiedene Risikofaktoren zugrunde, welche in der Patientenbetreuung multiprofessionell beachtet werden müssen. $\mathrm{Zu}$ den wichtigsten Risikofaktoren zählen Atemstörungen aufgrund von Schädigungen des Atmungssystems sowie Schonatmung aufgrund von Schmerzen und Immobilität. Ebenso fördern eine Schluckstörung, maschinelle Beatmung, verminderte Immunabwehr und Immobilität die Entstehung einer Pneumonie.

\section{Prophylaxe mit LISA}

Das Ziel der Pneumonieprophylaxe ist die Vermeidung einer Entzündung des Lungengewebes. Hierbei müssen die vorhandenen Risikofaktoren berücksichtigt und das patientenindividuelle Pneumonierisiko eingeschätzt werden. Zur Risikoeinschätzung kann die Atemskala nach Christel Bienstein genutzt werden. Nach erfolgter Risikoeinschätzung wird für den Patienten ein entsprechen-

Abb. 1: LISA: Ziele der Pneumonieprophylaxe

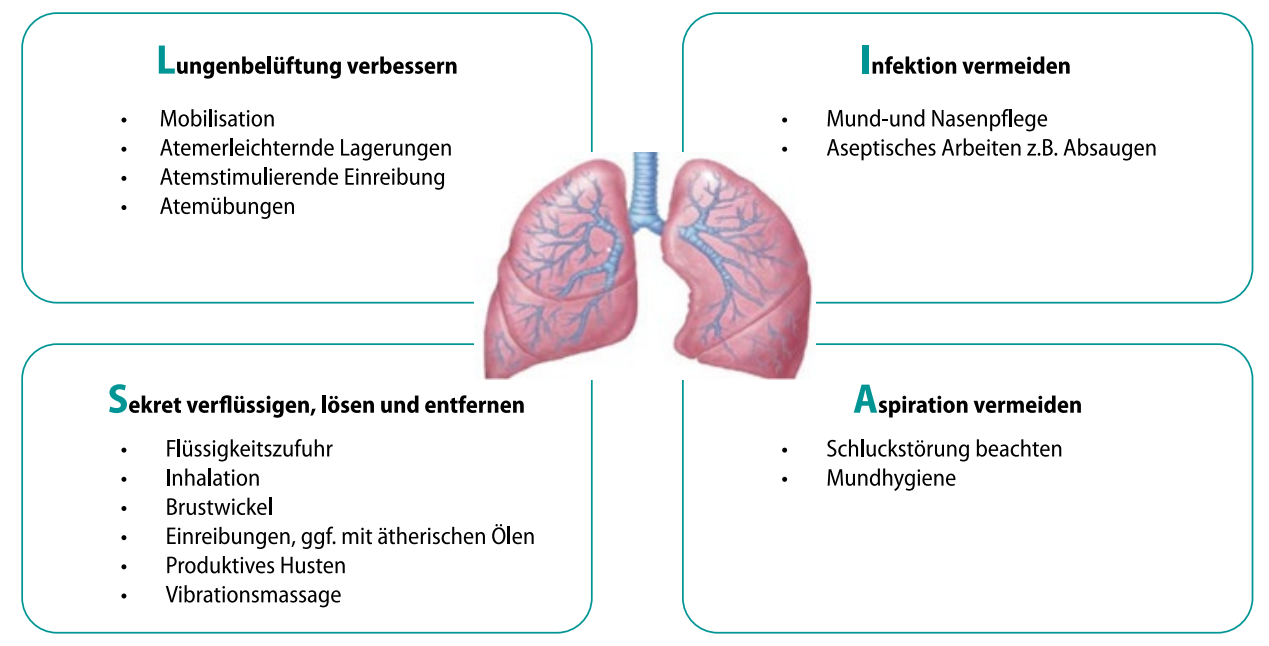




\section{† QUICK TIPPS}

\author{
_ Konsequent Pneumonierisikofaktoren erfassen \\ - Auf Grundlage der Risikoerfassung individuelle \\ Prophylaxemaßnahmen planen \\ _ Mobilität und Atemübungen in den täglichen \\ Pflegeprozess einbinden \\ - Patienten zu individuellen Prophylaxemaßnahmen \\ anleiten und schulen \\ - Alle Professionen zur Umsetzung einer ganzheitli- \\ chen/umfassenden Pneumonieprophylaxe \\ einbeziehen
}

der Therapieplan erarbeitet, welcher multiprofessionell und unter aktiver Einbeziehung des Patienten in der täglichen Betreuung umgesetzt wird.

Als Kernpunkte in der Pneumonieprophylaxe werden die LISAZiele formuliert:

_ Lungenbelüftung verbessern

- Infektion der Atemwege verhindern

- Sekretmobilisation fördern

_ Aspiration vermeiden

\section{Unspezifische Maßnahmen zur Pneumonieprophylaxe}

Neben der konsequenten Umsetzung der LISA-Ziele können weitere Prophylaxemaßnahmen eingesetzt werden, um das Pneumonierisiko zu senken und die Erkrankung zu vermeiden. Hierbei handelt es sich um Maßnahmen, welche die Pflegepersonen in den täglichen Pflegeprozess einfließen lassen. Ebenso erfolgt eine ressourcenorientierte Einbeziehung des Patienten sowie die Anleitung und Schulung zur selbstständigen Durchführung.

Mobilisation: Jede Form der Bewegung unterstützt und vertieft die Atmung - fördert somit die Belüftung aller Lungenabschnitte. Zusätzlich unterstützt die körperliche Aktivität die Mobilisierung des Bronchialschleims. Somit ist von zentraler Bedeutung, immobile Patienten entsprechend des Gesundheitszustandes zu mobilisieren, um eine konsequente Belüftung aller Lungenabschnitte anzuregen.

Atemerleichternde Lagerung: Neben der Mobilisation des Patienten kann durch eine atemerleichternde Lagerung das Pneumonierisiko für immobile und bettlägerige Patienten reduziert werden. Aufgrund der verschiedenen Lagerungen werden abwechselnd die einzelnen Lungenabschnitte belüftet und entsprechend besser durchblutet. Atemerleichternde Lagerungen können mehrmals täglich durchgeführt und die Positionen gewechselt werden. Zum Einsatz können u.a. die Oberkörperhochlagerung, Seitenlagerung, Halbmondlagerung in Rückenlage, VATI-Lagerung oder Dreh-Dehnlage kommen.

Weitere Maßnahmen: Dazu zählen ausreichende Flüssigkeitszufuhr (nach Rücksprache mit dem Arzt), Inhalation (nach Rücksprache mit dem Arzt), Anleitung zum produktiven Husten, gegebenenfalls
Absaugen, atemstimulierende Einreibungen, gegebenenfalls mit ätherischen Ölen (CAVE: Allergien), konsequente Mundhygiene und Aspirationsprophylaxe bei der Nahrungsaufnahme.

\section{Physiotherapeuten einbeziehen}

Neben den aufgeführten prophylaktischen Maßnahmen, wozu die Pflegepersonen den Patienten aktiv einbeziehen und anleiten, nehmen die Physiotherapeuten eine zentrale Rolle in der Durchführung der Pneumonieprophylaxe ein. Die Physiotherapeuten führen mit dem Patienten gemeinsam Übungen zur Verbesserung der Lungenbelüftung aus und unterstützen die Sekretlösung mit technischen Hilfsmitteln. Folgende Maßnahmen kommen zum Einsatz:

Atemübungen/Atemgymnastik: Hierbei wird die Belüftung aller Lungenabschnitte sowie die Selbstreinigungsmechanismen der Atemwege unterstützt und angeregt. Ebenso kann mit Atemübungen die richtige Atemtechnik erlernt werden.

Atmen gegen den Widerstand: Durch das Aufpusten von Luftballons, Wegpusten eines Wattebauschs (oder mit einem Strohhalm Flüssigkeit aufwirbeln) wird eine vertiefte Atmung angeregt (CAVE: Bei Patienten mit Lungenemphysem nur nach Arztrücksprache). Außerdem: Kontaktatmung, dosierte Lippenbremse ,Vibrationsmassagen und SMI Atemtrainer (Sustained Maximal InspirationTrainer) (z.B. Mediflo ${ }^{\oplus}$, Triflo ${ }^{\oplus}$ ).

III

\section{a PFLEGE EINFACH MACHEN}

Die Durchführung der Pneumonieprophylaxe ist in der täglichen Versorgung eine multiprofessionelle Aufgabe, welche den Patienten mit seinen Ressourcen und Problemstellungen in den Mittelpunkt stellt.

Entsprechend der Risikoerfassung werden mit dem Patienten individuelle Maßnahmen geplant und der Patient zur selbstständigen Durchführung angeleitet.

Durch die aktive Einbeziehung in die prophylaktischen Maßnahmen wird die Selbstständigkeit des Patienten gefördert und der Genesungsprozess positiv unterstützt.

\section{Literatur bei der Verfasserin}

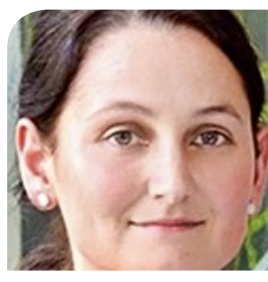

\section{Silke Müller}

Zentrumskoordination UniversitätsCentrum für Gesundes Altern

Universitätsklinikum Carl Gustav Carus Dresden Fetscherstraße 74

01307 Dresden

silke.mueller@ukdd.de

Ko-Autorin: Jana Luntz, Pflegedirektion 